\title{
Multiple frequency generation by bunched solitons in Josephson tunnel junctions
}

\author{
Lomdahl, P. S.; Sørensen, O. H.; Christiansen, Peter Leth; Scott, A. C.; Eilbeck, J. C.
}

Published in:

Physical Review B

Link to article, DOI:

10.1103/PhysRevB.24.7460

Publication date:

1981

Document Version

Publisher's PDF, also known as Version of record

Link back to DTU Orbit

Citation (APA):

Lomdahl, P. S., Sørensen, O. H., Christiansen, P. L., Scott, A. C., \& Eilbeck, J. C. (1981). Multiple frequency generation by bunched solitons in Josephson tunnel junctions. Physical Review B, 24(12), 7460-7462. https://doi.org/10.1103/PhysRevB.24.7460

\section{General rights}

Copyright and moral rights for the publications made accessible in the public portal are retained by the authors and/or other copyright owners and it is a condition of accessing publications that users recognise and abide by the legal requirements associated with these rights.

- Users may download and print one copy of any publication from the public portal for the purpose of private study or research.

- You may not further distribute the material or use it for any profit-making activity or commercial gain

- You may freely distribute the URL identifying the publication in the public portal 


\title{
Multiple frequency generation by bunched solitons in Josephson tunnel junctions
}

\author{
P. S. Lomdahl, O. H. Soerensen, and P. L. Christiansen \\ Laboratory of Applied Mathematical Physics, The Technical University of Denmark, DK-2800 Lyngby, Denmark
}

A. C. Scott

Center for Nonlinear Studies, Los Alamos Scientific Laboratories, Los Alamos, New Mexico 87545

J. C. Eilbeck

Department of Mathematics, Heriot-Watt University, Edinburgh EH14 4AS, United Kıngdom

(Received 24 August 1981)

\begin{abstract}
A detailed numerical study of a long Josephson tunnel junction modeled by a perturbed sineGordon equation demonstrates the existence of a variety of bunched soliton configurations. Thus, on the third zero-field step of the $V-I$ characteristic, two simultaneous adjacent frequencies are generated in a narrow bias current range. The analysis of the soliton modes provides an explanation of recent experimental observations.
\end{abstract}

The fundamental concept of soliton excitations was used by Fulton and Dynes ${ }^{1}$ to explain the appearance of zero-field steps (ZFS) in the voltage-current characteristic of long Josephson junctions. Thus the simultaneous excitation of $N$ solitons gives rise to the $N$ th ZFS. Recent numerical studies ${ }^{2,3}$ have demonstrated that two solitons can travel in a congealed state ("bunched fluxons"). The results are in agreement with experimental measurements and ascertainments made by Dueholm et al. ${ }^{4}$ These authors also reported on measurements for higher-order ZFS's showing a fine structure in the form of branching and small hysteresis loops accompanied by simultaneous emission of signals at two frequencies in the $X$ band with a separation of $\sim 50 \mathrm{MHz}$. This is illustrated in Fig. 1 obtained with a bias point on the third ZFS. The experimental recordings show the second harmonic of the fundamental frequency $f_{1}=u / 2 L$ where

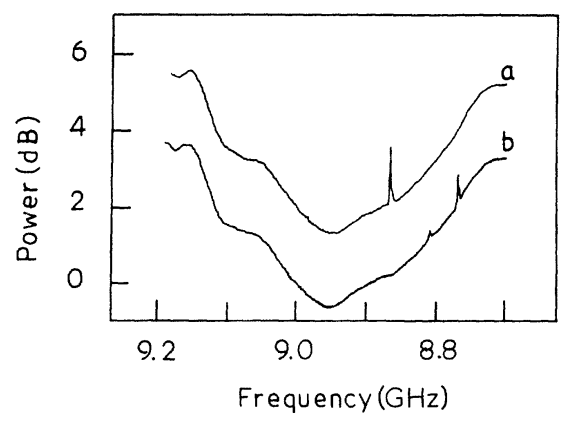

FIG. 1. Frequency display of experimentally observed microwave radiation from niobium oxide-lead Josephson junction. Trace $a$ and trace $b$ are obtained for $\gamma=0.14$ and 0.115 , respectively. $u$ is the soliton velocity and $L$ is the length of the junction. Trace $(a)$ shows a single frequency signal as observed on the major portion of the step and trace $(b)$ shows the appearance of two simultaneous signals observed in a narrow range of bias currents. ${ }^{5}$ It was suggested ${ }^{4}$ that this complex behavior could be explained by different bunching configurations of the solitons.

In this Communication we demonstrate that the different bunching configurations result from the perturbed sine-Gordon model ${ }^{6}$ given by

$$
\phi_{x x}-\phi_{t t}-\sin \phi=\alpha \phi_{t}-\beta \phi_{x x t}-\gamma \text {. }
$$

Here $\phi$ is magnetic flux normalized to $\hbar / 2 e, x$ is longitudinal distance normalized to the Josephson penetration depth $\lambda_{J}$, and $t$ is time normalized with respect to the Josephson "plasma" frequency $\omega_{0}$." The $\gamma$ term $^{8}$ represents a uniformly distributed bias current normalized to the maximum zero voltage (Josephson) current $I_{0}$. The terms in $\alpha$ and $\beta$ represent quasiparticle loss and surface resistance loss, respectively. Throughout the computations we have maintained "open-circuit" boundary conditions, i.e., $\phi_{x}(0, t)=\phi_{x}(L, t)=0$. For the junction discussed here $\alpha=0.05, \beta=0.02$, and $L=6$ as in Ref. 3 . As initial conditions we used soliton configurations consisting of either (i) three solitons moving with equal velocities or (ii) two solitons moving with the same velocity and an antisoliton moving in the opposite direction, leading to the third ZFS in both cases. The velocities were chosen sufficiently high to obtain soliton confinement on the junction by Lorentz contraction. The resulting steady-state oscillations were usually independent of the details of the initial conditions. We have used an implicit finite difference scheme with a corrector loop for the non-

7460 C1981 The American Physical Society 

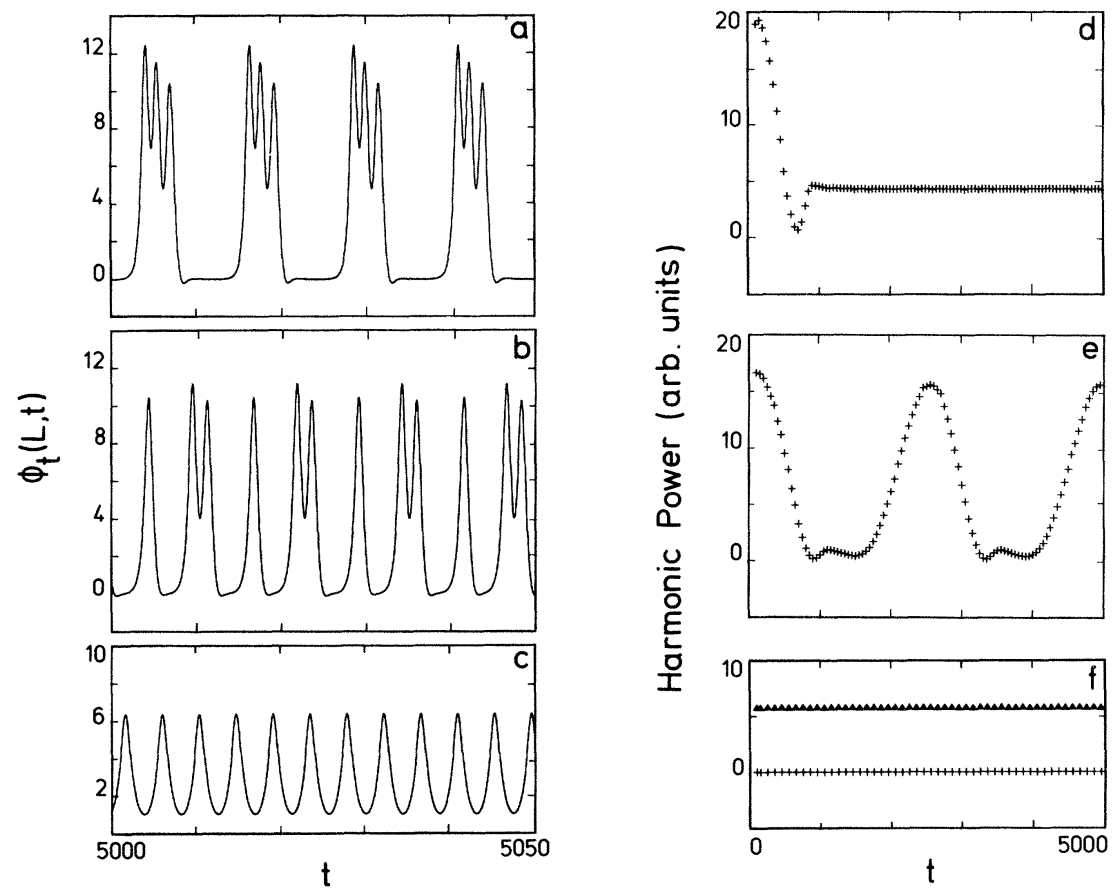

FIG. 2. Numerical computations of $\phi_{t}(L, t)$ showing different soliton modes for (a) $\gamma=0.42$, (b) $\gamma=0.4$, and (c) $\gamma=0.2$. The corresponding second $(+)$ and third $(\Delta)$ harmonic powers vs time are shown in $(d),(e)$, and $(f)$.

linear term and averaging of the second space derivative over two time steps. The time and space steps used were 0.025 and 0.05 , respectively. The accuracy of our computations was checked by halving time and space steps.

Typical numerical results for $\phi_{t}$ at one end of the junction are shown in Figs. 2(a) -2(c) after 5000 time units for different values of $\gamma$. In Fig. 2(a) $(\gamma=0.42)$ the three solitons propagate with velocity $u$ in a bunch producing the fundamental frequency $f_{1}=u / 2 L$. For decreasing $\gamma$ 's [Fig. 2(b), $\gamma=0.4$ ] we enter a narrow region in which two solitons remain bunched and the third travels alone with a slightly different velocity $(u+\Delta u)$ giving rise to two frequencies with a separation $\Delta f=\Delta u / 2 L$ [demonstrated below in Fig. 2(e)]. Approximately one half period of the time evolution in this case is presented in Fig. 3. For a further decrease in $\gamma$ the bunching configuration of Fig. 2(a) is recovered. This configuration then gradually changes into the uniform oscillation shown in Fig. 2(c) $(\gamma=0.2)$. The fundamental frequency in this mode equals $3 f_{1}$. In Figs. 2(d)2 (f) we show the corresponding harmonic power content of $\phi_{t}(L, t)$ as function of time. We have analyzed 100 subsequent time intervals of length 50 time units between $t=0$ and $t=5000$. In all three cases initial conditions of type (ii) were used. Figure 2(d) shows the second harmonic for $\gamma=0.42$. After a transient the second harmonic assumes a constant value corresponding to the formation of a stable bunch of three solitons [cf. Fig. 2(a)]. In Fig. 2(e) $(\gamma=0.4)$ the three-soliton bunch is unstable - the single soliton is never captured by the two-soliton bunch. Owing to the coexistence of two slightly different frequencies on the junction, the second harmonic is seen to vary periodically in time with a frequency $23 \mathrm{MHz}$, in good agreeement with the experi-

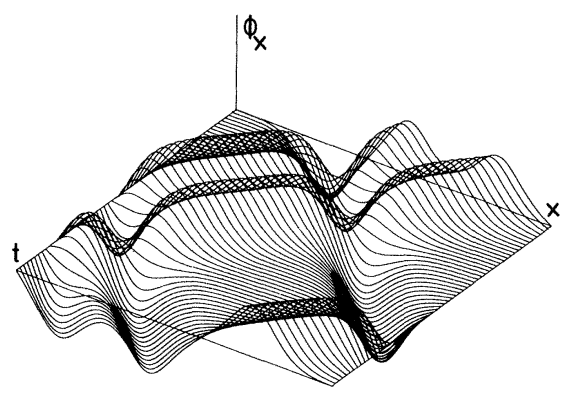

FIG. 3. A $2+1$ bunched soliton solution in Eq. (1) with $\alpha=0.05, \beta=0.02, \gamma=0.4, L=6$. Approximately one-half period of $\phi_{x}(x, t)$ is plotted for $5000 \leqslant t \leqslant 5006$. 


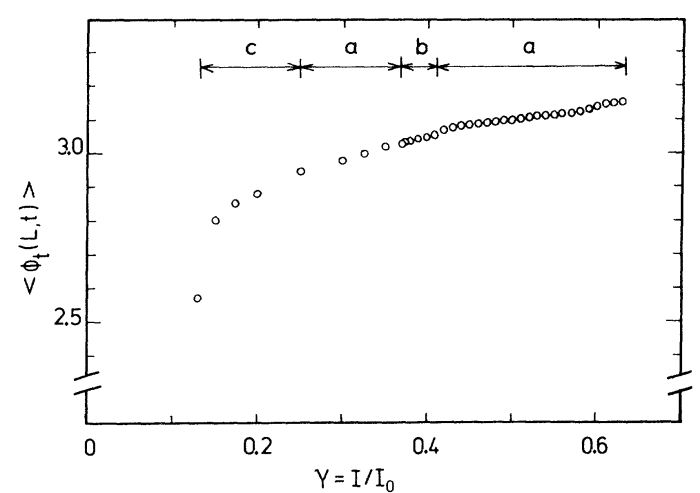

FIG. 4. Calculated third ZFS $\left[\left\langle\phi_{t}(L, t)\right\rangle\right.$ vs $\left.\gamma\right]$. The $\gamma$ intervals $a-c$ correspond to soliton configurations shown in Figs. 2(a) $-(2 \mathrm{c})$.

mental result shown in Fig. 1. The maximum power is obtained with the relative positions of the solitons shown in Fig. 2(b). Figure 2(f) shows the vanishing second harmonic (crosses) and the finite third harmonic (triangles), corresponding to $3 f_{1}$ for $\gamma=0.2$.

Finally, Fig. 4 shows the calculated time average of
$\phi_{t}(L, t)$, proportional to the dc junction voltage, versus $\gamma$, proportional to the bias current. Hence the figure is the calculated third ZFS in the $V-I$ characteristic of the junction. Experimentally ${ }^{4,5}$ structures were often observed in the higher-order ZFS's $(N \geqslant 4)$ which were interpreted as manifestations of transitions between different multisoliton modes. Here, the apparent structure in the ZFS which is barely visible in Fig. 4 at the transition from the three-soliton bunch [Fig. 2(a)] to the $(2+1)$-soliton configuration [Fig. 2(b)] is of the same order of magnitude as our numerical resolution.

We conclude that our analysis of the soliton modes explains the appearance of two simultaneous frequencies in agreement with experimental observations.

It is our pleasure to thank Physics Laboratory I at The Technical University of Denmark and the authors of Ref. 4 for providing the unpublished measurments in Fig. 1 and R. D. Parmentier for many helpful discussions. The support by the Danish Council for Scientific and Technical Research, the Danish Natural Science Research Council, and Otto Mønsteds Fond is acknowledged.
${ }^{1}$ T. A. Fulton and R. C. Dynes, Solid State Commmun. 12, 57 (1973).

${ }^{2}$ S. N. Erné and R. D. Parmentier, IEEE Trans. Magn. 17, 804 (1981)

${ }^{3}$ P. L. Christiansen, P. S. Lomdahl, A. C. Scott, O. H. Soerensen, and J. C. Eilbeck, Appl. Phys. Lett. 39, 108 (1981).

${ }^{4}$ B. Dueholm, O. A. Levring, J. Mygind, N. F. Pedersen, O. H. Soerensen, and M. Cerillo, Phys. Rev. Lett. 46, 1299 (1981).
${ }^{5}$ Unpublished results of the authors of Ref. 4. The simultaneous appearance of two frequencies was more pronouced on the higher ZFS's. However, for convenience the numerical simulation is carried out for the third ZFS

${ }^{6}$ A. C. Scott, F. Y. F. Chu, and S. A. Reible, J. Appl. Phys. 47, 3272 (1976)

${ }^{7}$ The junction on which the results in Fig. 1 were obtained had $\lambda_{J}=156 \mu \mathrm{m}$ and $\omega_{0}=5.8 \times 10^{10} \mathrm{~s}^{-1}$.

${ }^{8}$ W. J. Johnson and A. Barone, J. Appl. Phys. 41, 2958 (1970). 